\title{
Research on Position-Based Routing Protocol HVSR in Ad Hoc Networks
}

\author{
Hou Zhan-sheng ${ }^{1, a}$, Xu Min ${ }^{2, b}$, Liu Wei, ${ }^{3, c}$, Wu Wei-nong ${ }^{4, d}$, BaoXing-chuan ${ }^{5, e}$, \\ Wang Gang ${ }^{6, f}$, Pen Lin ${ }^{7, h}$, Han Hai-yun ${ }^{8, i}$, Zhu Liang ${ }^{9, j}$ \\ ${ }^{1}$ State Grid Smart Grid Research Institute; Nanjing China; 210003; \\ ${ }^{2}$ State Grid Smart Grid Research Institute; Nanjing China; 210003; \\ ${ }^{3}$ State Grid Chongqing Electric Power Company Chong Qing; 401121;

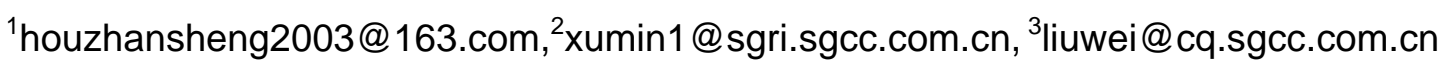

\begin{abstract}
Keywords: Ad Hoc Networks, Position-Based Routing,Forwarding Hole, Scalability
Abstract: Ad Hoc network is a multi-hop temporary self-organized system, which consists of a set of mobile terminal with the radio devices. Each mobile terminal is both a host and router, performing route setup and packet forwarding according to a routing algorithm. Location-based routing algorithm eliminates the limitations of topology-based routing algorithm by the additional location information. It not only has higher reliability and better adaptability than dynamic topology, but also has low overhead, high efficiency and scalability.This paper studied the location-based services of grid routing protocol (GLS), and analysed its strengths and weaknesses, then proposed a location-based routing protocol HVSR. Compareing with the GLS, It has Lower overhead, higher efficiency and reliability.
\end{abstract}

\section{Introduction}

Mobile Ad Hoc Network is a dynamic volatile network [1], were blocked or under obstructions in the signal, especially a sharp increase in the number of nodes and node intermittently connected to the network, the existing Ad Hoc routing protocol will be drastically reduced reliability, a sharp decline in performance. Therefore, the design of low cost, high efficiency, high reliability and scalability [2] based on the location information routing protocol, it is of great significance for Ad Hoc network applications.

\section{Location information based routing protocol HVSR}

\subsection{GLS routing protocol works}

GLS (Grid Location Service) [4] was first proposed by J.Li, GLS routing protocol is a routing protocol based on the location information, the use of locator service routing positioning, packet forwarding, location information maintained routing protocol.

The basic idea GLS is a hierarchical location service method, and the first sub-grid technology and the location information service combination. In GLS, which will be divided into a grid network topology. In this layered structure, the first $n$ layer 4 the next layer comprises a grid (i.e., layer n-1) of the grid, each grid contains a second-order-order four grids 1, each of the third-order network cell contains four second-order grid. HELLO packet broadcast regularly to every point in each of the first-order grid, and location information of all the points within the first-order grid storage node in the neighbor table. Each node maintains multiple locations throughout the network server, the location of the server is not specified, but a distributed algorithm. After a distributed algorithm, each node in the number of local servers from its more recent, but it is far away from the small number of servers. As a location server number will increase with the order of the grid is reduced. Each node may have other nodes as a location server, location server architecture and also no difference between an ordinary node. 


\subsection{HVSR routing algorithm improved the GLS}

\subsubsection{Protocol Model}

HVSR (Horizontal and Vertical Strip Routing) is a routing protocol based on location information, which is a fault-tolerant distributed [3] location information protocol. It has a high efficiency, reliability and robustness. And GLS common , HVSR agreement is the network area into a square grid area [4] [5], but the difference is that it is only one layer, then put the grid area is divided by horizontal and vertical band to band like area, each node location information will be stored on all nodes within it in the strip. And when the server location update, HVSR using aggregation technology, each location update package gathered location information and a number of nodes simultaneously update multiple location server. In addition, aggregate updates are synchronized, in a particular area, there will only be a boundary node initiating aggregation update, so there is no repeat of the update package. In HVSR each strap area, gathered update package is initiated by the boundary nodes and regularly along the strip area initiate a location update. 1 illustrates the point $S$ and the neighbor taking it in the strip area graph. Node A network region, $\mathrm{W}, \mathrm{X}$ and $\mathrm{Y}$ are the border strip area network nodes.

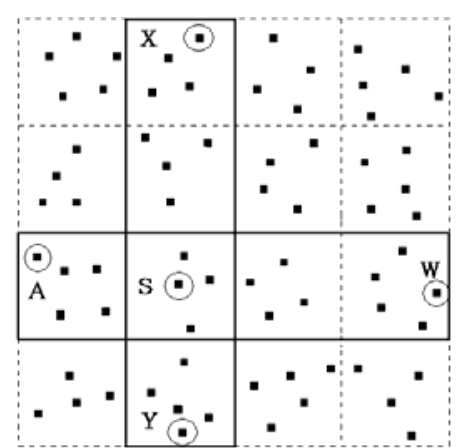

Figure 1 Strip-shaped region and the terminal node of the network of distribution

\subsubsection{Principle agreement}

Similar GLS, HVSR mainly composed of four parts [4]:HELLO packets, a location update, location query, data forwarding.

HELLO packets. Location HVSR routing protocol neighbor node coordinates by sending HELLO packets way to locate, HELLO packets containing an identifier and location information for the current node. Before starting the implementation of the algorithm, since the node by locating the server know your location, the next step is the exchange of HELLO messages between neighboring nodes. When the node receives the neighbor node HELLO packet, the packet node location information and node identifier information belongs strip area and the effective time written into its cache, while record time at the message reception and to that end the sending node assigned an identity. Principles for allocating identity is ' 0 ' for the current node identifier, strip-shaped region if the current node is located in $\mathrm{m}$ neighbor, put $1 \sim \mathrm{m}$ this $\mathrm{m}$ integers $\mathrm{m}$ randomly assigned to its neighbor node as its identity, so both to ensure they do not have to complete the localization algorithm for all the nodes are assigned a global identity, not only reduces the complexity of the algorithm, but also enhance the robustness and reliability of routing protocols. HELLO messages sent by the nodes set a timer, time hello_timeout, every node performs a cycle hello_timeout HELLO packets. If the node is not received within 2 hello_timeout some neighboring nodes HELLO packet, the nodes are no longer considered to be a neighbor of this node. Thereby to remove those nodes from his neighbor nodes in the domain. 


\section{Location Update}

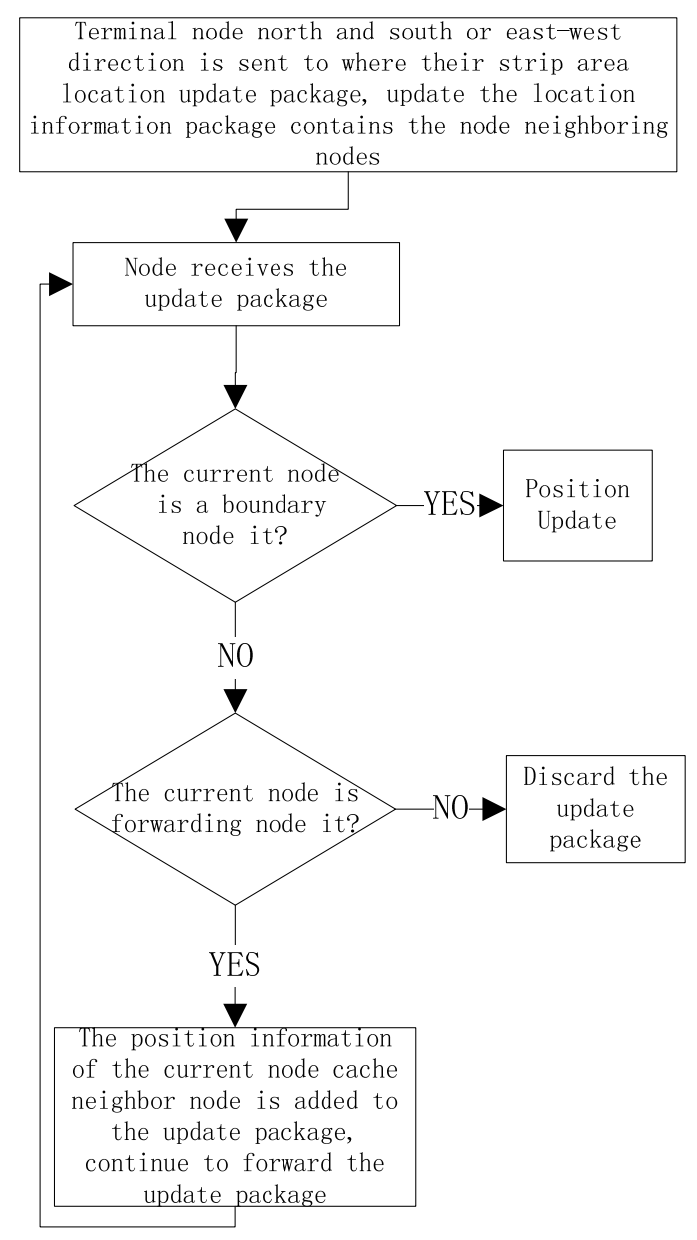

Figure 2 Location Update flow chart

And GLS biggest difference is that, when HVSR location server update algorithm used protocols, update packages and query the package data structures and types are as follows:

Node identifier: id;

Horizontal and vertical banded zone identifier: hid, vid;

Direction of the strip-shaped region domain: Array Strip [i] where $i \in\{$ north $=0$, south $=1$, west $=2$, east $=3\}$;

The current node identification: this.id, next hop node identification: next.id;

Update package is effective: to strip_update_timeout, namely an update cycle;

Effective time query messages: set to discovery_timeout, which is a query cycle;

Neighbor domain of the current node: node within radio range and located node resides on a strip-shaped region that $\{\mathrm{n} \in$ neighbors $\mid$ n.hid $=$ sid $\vee$ n.vid $=$ sid $\}$.

Select the next hop node: We select the domain from the current node neighbor farthest node as its next hop node, the literature [2] shows that this approach is the most effective, the node to wireless transmission furthest away from their domain node as the next hop node.

Location Query. HVSR and GLS Another big difference is that the destination node location name lookup algorithm used, position hierarchical routing protocol GLS target node query using the "closest node" It's in each layer [5] as the location server, and then click Query until you find the destination node. In HVSR the current node querying the target node is in their area in a horizontal or vertical strip carried out. Location query protocol stored in the node strip [i] and the neighbor node identification information to locate the target node. Location query flow chart shown in Figure 3. 


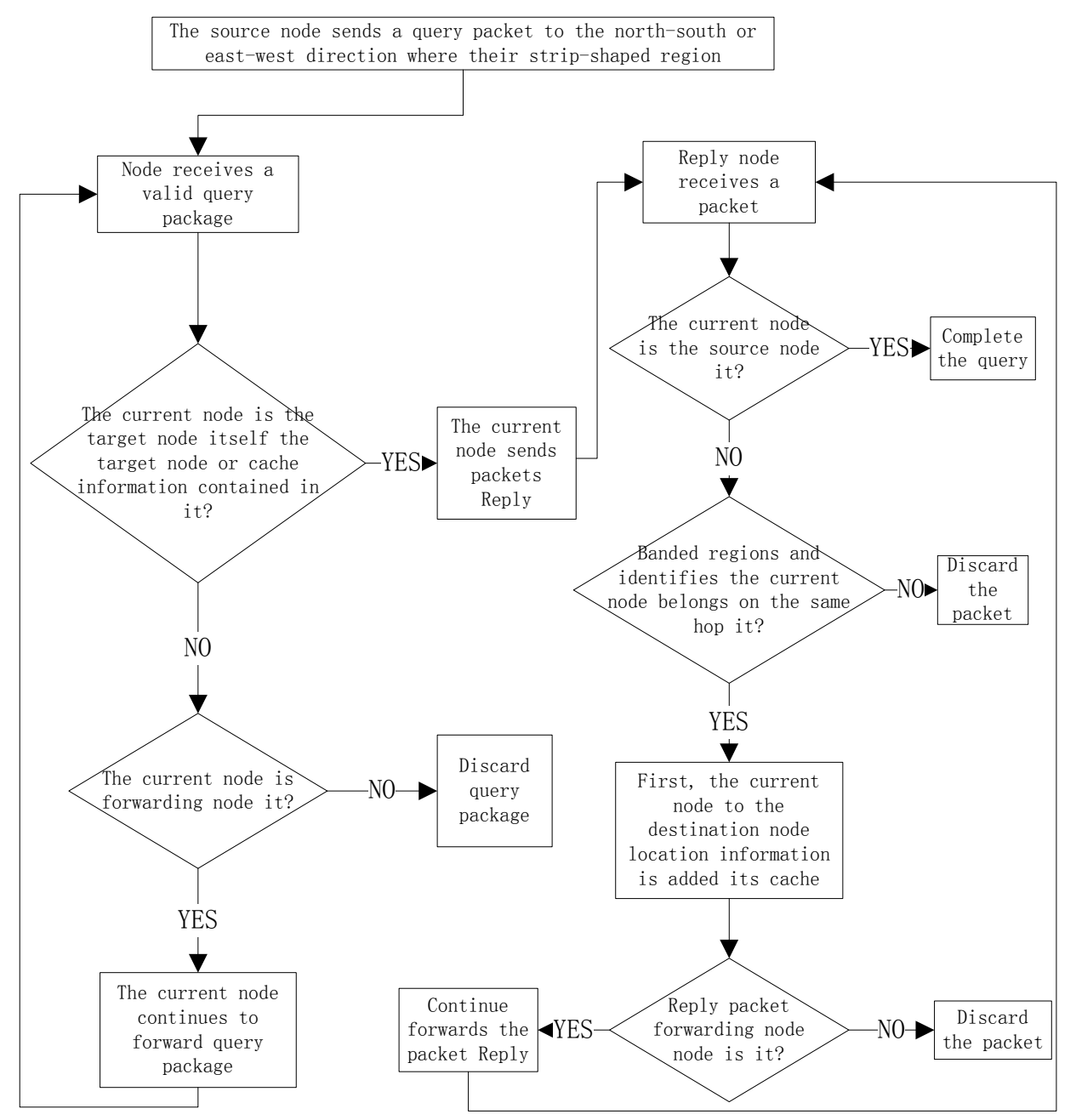

Figure 3 position search algorithm flowchart

Data Forwarding. HVSR data forwarding algorithm using similar GLS, shown in Figure 4, the source node $\mathrm{S} \mathrm{m}$ forward packets to the target node T. First, the source node S first position in the implementation of local information, query their own cache information table and location information table, if the location information to find the target node, then the direct use of geographic forwarding algorithm sends packets to the destination node, or else start position search algorithm, When you find the location of the target node $\mathrm{T}$, and then forwarding algorithm execution location.

Flanking route lookup. As shown in Figure 5, In this case, in order to make the source node S sending a packet to the target node $\mathrm{T}$, we use flanking route lookup method to offset the impact caused by the forwarding vulnerabilities. If the data packet when the source node $\mathrm{S}$ sent through a square grid area and $\mathrm{b}$ forwards failed to reach the target node $\mathrm{T}$, shown in Figure 5 , the node $\mathrm{X}$ receives the packet, but could not find the square grid area $b$ When forwarding node, this time, the node $\mathrm{X}$ will start secondary route lookup mechanism to reposition the location information of the target node $\mathrm{T}$, first send a query packet to the horizontal direction where their strip-shaped region, node $\mathrm{Y}$ receives a query packet, because $\mathrm{Y}$ node does not know the location information of the target node $\mathrm{T}$, it continues to forward the packet, when the node $\mathrm{Z}$ receives the query packet node $\mathrm{Z}$ will know the location information of the target node $\mathrm{T}$ reply by the response packet to the node $\mathrm{X}$, node $\mathrm{X}$ then The response packet is forwarded to the source node $\mathrm{S}$. At this time again the source node $\mathrm{S}$ sending a packet destination node $\mathrm{T}$, the final packet reaches the destination node $\mathrm{T}$, thereby completing the data transmission. 


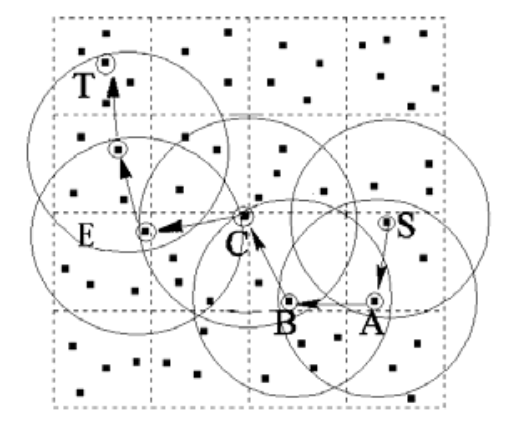

Figure 4 packet forwarding path map

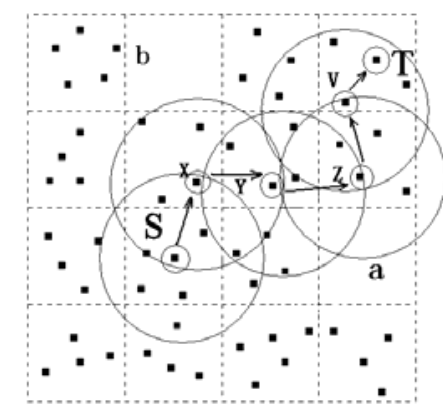

Figure 5 grid area a and $\mathrm{b}$ are present forwarding Bypass Vulnerability

\section{HVSR simulation results compared with GLS}

In this paper, as a simulation tool NS2 simulation platform is built for the NS 2.28 installed version of the emulator, use CMU wireless expansion modules [7] simulation experiment under Red Hat Linux 9.0 systems, Experiments in the area of the region $1.0 \times 1.0 \mathrm{~km}^{2}, 1.4 \times 1.4 \mathrm{~km}^{2}$, $1.7 \times 1.7 \mathrm{~km}^{2}, 2.0 \times 2.0 \mathrm{~km}^{2}, 2.3 \times 2.3 \mathrm{~km}^{2}$, The total number of individual network nodes were $100,200,300,400,500$ region. Node density is $100 \mathrm{~km}$, nodes per square uniformly distributed random wireless transmission radius of each node is $250 \mathrm{~m}$, the width of the strip-shaped region of $200 \mathrm{~m}$. HELLO messages sent every $2 \mathrm{~s}$, allow loss frequency is 2; location update package strip_update_timeout set to 10s; discovery_timeout location query packet and retransmissions_timeout set to 2s, query packet retransmission packet no more than 2 times.

\subsection{Query success rate}

Figure 6 shows a comparison of the query success rate, which, GLS-100 and GLS-200, respectively, GLS location update threshold of $100 \mathrm{~m}$ and $200 \mathrm{~m}$. When the smaller network size, HVSR and GLS have very high reliability can be achieved, but with the increase of network size, HVSR have higher reliability than the GLS. Especially in large-scale networks, HVSR query success rate than the GLS-100 and GLS-200 were much higher.

One reason is that, in HVSR, the terminal node initiates a location update frequency is high (the experiment is set to 10s). Distance GLS location update frequency and the update package required transmission related. Each mobile node $\left(2^{\mathrm{i}-2 *} \mathrm{~d}\right)$ distance its i-layer location server updates the location information, $d$ is specially selected from the threshold. In this way, the update frequency and node mobility rate node is directly proportional to the distance between the location server and node. When the network size increases, the GLS, the remote location server redundancy is small and long time to update the location information.

\subsection{Comparison of Reliability}

HVSR relative GLS biggest advantage is its excellent reliability and fault tolerance [6]. We will HVSR and GLS comparison, $2.3 \mathrm{~km} * 2.3 \mathrm{~km}$ network area, the experiment to keep the number of connections to node 400 .

As can be seen from Figure 7, HVSR query success rate and reliability with network nodes unstable moderately lower percentage increases. The GLS query success rate and reliability are drastically reduced when the proportion of unstable nodes is 50\%, GLS query success rate dropped to 75 percent; when all nodes are unstable node, GLS query success rate drop 52\%. Forwards similar reliability. 


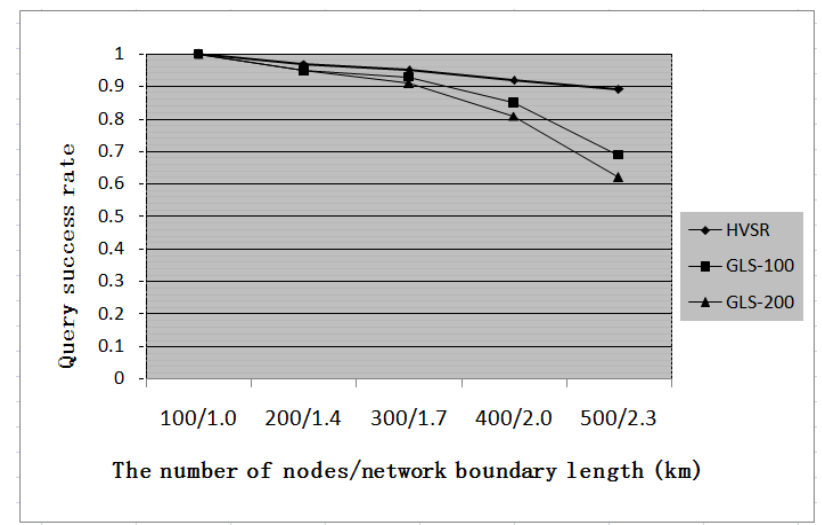

Figure 6 Compare GLS and HVSR query success rate

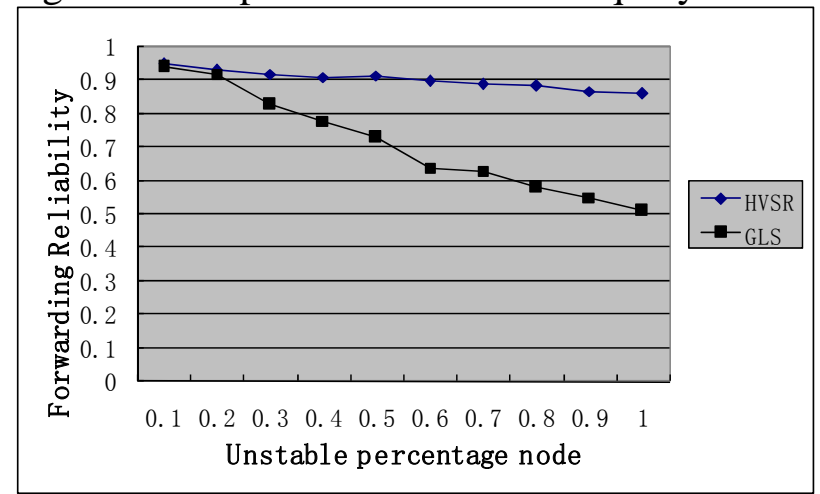

Figure 7 Reliability unstable relationship percentage and forwarding node

\section{Conclusion}

This paper studies the GLS routing protocol, analyzes its strengths and weaknesses, on the basis of improved results-based routing protocol HVSR location information through simulation experiments and GLS HVSR performance comparison. Shows the query success rate and reliability, etc. HVSR forwarding routing protocol than GLS has better performance.

\section{Reference}

[1] Wang Haitao, Zhao Zhifeng, MI Zhi Chao, LI Ning. "Ad Hoc network technology." People Post Press, 2005.

[2] The agreement and its properties .MANET routing Xiao Shucheng, Tangxue Wen, Wang Kang Computer Engineering and Design, 2004,25 (7): 1133-1136.

[3] L.Blazevic and J.Y.Le Boudec and S.Giordano, A Location-Based Routing Method for Mobile Ad Hoc Networks,IEEE Transactions on Mobile Computing, pp. 97-110, 2005.

[4]J.Li,.Jannotti,D.De Couto,D.Karger and R.Morris,A scalable Location service for geographic ad-hoc routing,in: Proceedings of The 6th ACM International Conference on Mobile Computing and Networking (MobiCom’00) (August2000) pp.120-130.

[5] Roie Melamed and Idit Keidar and Yoav Barel, A fault-tolerant and efficient ad-hoc routing protocol,Proceedings of the 2005 24th IEEE Symposium on Reliable Distributed Systems,October26-28,2005.

[6] S. Miry, A. A. Pirzada, M. Portmann. HOVER: Hybird on-demand Distance Vector Routing for Wireless Mesh Networks. Australian Computer Society Inc.2008: 63-71.

[7] Xu thunder, Pombo, Zhao Yao. "NS and network simulation." People Post Press .2003 (11). 